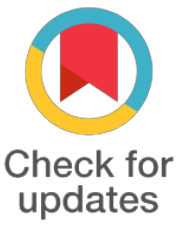

*For correspondence:

thilathy@upm.edu.my

Competing interests: The authors declare that no competing interests exist.

Received: 2017-06-30

Accepted: 2017-07-25

Published: 2017-09-05

Copyright The Author(s) 2017. This article is published with open access by BioMedPress (BMP).

This article is distributed under the terms of the Creative Commons Attribution License (CC-BY 4.0) which permits any use, distribution, and reproduction in any medium, provided the original author(s) and the source are credited.

\section{Comparative characterization of murine Bone marrow mesenchymal stem cells cultured using two different supplements}

\author{
Kwan Liang Lye ${ }^{1}$, Nurfazilahyusnitafitrah Sulaiman ${ }^{1}$, Sharmili \\ Vidyadaran ${ }^{2,3}$ Norshariza Nordin1,3, Karuppiah Thilakavathy ${ }^{1,3}$ \\ ${ }^{1}$ Department of Biomedical Sciences, Faculty of Medicine and Health Sciences, Universiti Putra \\ Malaysia, 43400 UPM Serdang, Selangor, Malaysia. \\ ${ }^{2}$ Department of Pathology, Faculty of Medicine and Health Sciences, Universiti Putra Malaysia, \\ 43400 UPM Serdang, Selangor, Malaysia. \\ ${ }^{3}$ Genetics and Regenerative Medicine Research Centre, Faculty of Medicine and Health \\ Sciences, Universiti Putra Malaysia, 43400 UPM Serdang, Selangor, Malaysia.
}

Abstract

Preclinical studies on mesenchymal stem cells (MSC) have allowed the cells to be considered as a promising candidate for cellular therapy. The mouse is the most widely used species for studying the characteristics of MSC. In recent years, conflicting data were reported regarding growth kinetics, surface marker profile, differentiation capacity, genetic instability or malignant transformation and so forth, that may be a result of a range of factors. One of the factors probably is the culture medium formulation. Here we have made a comparative characterization of bone marrow-derived mesenchymal stem cells (mBM-MSC), under the same experimental conditions, cultured using two common supplements, fetal bovine serum (FBS) and MesenCultTM Stimulatory Supplement (MSS). mBM-MSC isolated from the tibias of $\mathrm{C}_{57 \mathrm{BL} / 6}$ mice were cultured and expanded in Dulbecco's Modified Eagle's Medium supplemented with either $15 \%$ FBS or $15 \%$ MSS. Clonogenic potential, population doubling time, immunophenotyping, differentiation immunosuppression potentials and chromosome analysis of early and late passage of mBM-MSC were assessed.

The findings showed that the immunophenotype and differentiation potential of mBMMSC were similar when cultured using these supplements irrespective of passages. Variations were seen in clonogenic, growth, proliferation rate and immunosuppression potential of the mBM-MSC. This study also revealed that prolonged culture will disrupt their genetic stability regardless of the supplements used. The genetically mutated mBM-MSC were also found to maintain their stemness characteristics and immunosuppression potential.

In conclusion, culture medium formulation causes variations in the cultured MSC and may influence downstream investigation findings.

Keywords

Funding

References 